\title{
Fatores de risco e patogênese das neoplasias malignas epiteliais de ovário: revisão de literatura
}

Risk factors and pathogenesis of malignant epithelial ovarian neoplasm: a literature review

\author{
Caroline Maria Ristow' ${ }^{1}$ Célia Toshie Yamamoto², Mariana Fávaro
}

\section{Resumo}

O carcinoma epitelial de ovário é a segunda neoplasia mais comum do trato genital feminino, somando 5\% das neoplasias nas mulheres, segundo dados internacionais. No Brasil, o câncer de ovário ocupa o terceiro lugar entre as neoplasias femininas. Por sua prevalência e desenvolvimento silencioso, torna-se importante discorrer sobre a patogênese desse carcinoma, que se não detectado precocemente produz elevada mortalidade, apesar dos avanços no manejo da doença avançada. Esse estudo objetivou uma análise crítica de pesquisas previamente publicadas em periódicos indexados nas bases de dados Medline e Scielo a respeito do tema abordado. Existem várias teorias em relação à carcinogênese em tecido epitelial ovariano. Com base nessas, pesquisou-se informaçóes sobre os fatores protetores e entre eles estão paridade, amamentação, uso de anticoncepcionais orais, menarca tardia e menopausa precoce, procedimentos cirúrgicos, como histerectomia e ligação tubária. Os principais fatores relacionados ao aumento do risco são uso de drogas indutoras de ovulação, altos níveis de estrógenos e androgênios, fatores genéticos e ambientais. O perfil epidemiológico associado à identificação de fatores de risco continua sendo o melhor recurso de que dispomos para uma detecção precoce dessa patologia, uma vez que até o momento não há programas de controle para os fatores de risco citados e o rastreamento, através de marcadores séricos (CA-125) e ultra-sonografias (transvaginal e pélvica), é preconizado para mulheres com antecedentes familiares de câncer ovariano genético.

Palavras-chave: Neoplasias ovarianas, Fatores de risco, Patogênese, Epidemiologia, Revisão.

${ }^{1}$ Doutoranda da Faculdade Evangélica do Paraná (FEPAR).

${ }^{2}$ Doutora e Professora Assistente da Disciplina de Ginecologia e Obstetrícia da Faculdade Evangélica do Paraná.

Trabalho realizado na Faculdade Evangélica do Paraná - Disciplinas de Clínica Cirúrgica (Oncologia) e de Ginecologia e Obstetrícia.

Enderȩ̧o para correspondência: CMR. Rua Miguel Abrao, 90. Portão-Curitiba (PR). Cep: 81070-140. E-mail: carol_ristow@yahoo.com.br. 


\section{Abstract}

Epithelial carcinoma of the ovary is the second most common female genital tract neoplasm, representing 5\% of neoplasms in women, according to international data. In Brazil, ovarian cancer occupies third place among neoplasms in females. Due to its prevalence and silent evolution, it is important to study the pathogenesis of this carcinoma, which leads to high mortality if not detected early, despite progress in management of the advanced disease. The current study aimed at a critical analysis of published previously research from indexed periodicals in the Medline and Scielo databases. There are various theories on carcinogenesis in the ovarian epithelial tissue, based on which we searched for information on protective factors, including: parity, breastfeeding, use of oral contraceptives, late menarche and early menopause, and surgical procedures such as hysterectomy, and tubal ligation. The principal factors related to increased risk are: use of ovulation-inducing drugs, high estrogen and androgen levels, and genetic and environmental factors. The epidemiological profile and identification of risk factors are still the best available resources for early detection of this disease, since thus far there are no programs to control the abovementioned risk factors. Screening with serum markers (CA-125) and ultrasound (transvaginal and pelvic) is recommended for women with a family history of ovarian cancer.

Key words: Ovarian carcinomas, Risk factors, Pathogenesis, epidemiology, Literature review.

\section{INTRODUÇÃO}

A neoplasia maligna epitelial de ovário é o segundo câncer mais comum do trato genital feminino, somando cerca de 5\% das neoplasias malignas em mulheres, segundo dados internacionais ${ }^{1}$. Os dados brasileiros de 1999 do Instituto Nacional do Câncer (INCA) ${ }^{2}$ e os de 1982, da Campanha Nacional de Combate ao Câncer ${ }^{3}$, situam as neoplasias malignas do ovário como sendo a terceira em ordem de incidência, entre aquelas que acometem os órgãos genitais femininos, ficando abaixo das lesóes do colo e do corpo do útero ${ }^{4}$. Nos Estados Unidos, a cada 70 mulheres uma é acometida pela neoplasia maligna epitelial de ovário, sendo que para 2004 eram esperados 25.580 novos casos e 16.090 óbitos por esta patologia ${ }^{5}$. A incidência dessa patologia é maior em países mais industrializados e de níveis sócioeconômicos maiores ${ }^{4}$, com exceção do Japão ${ }^{6}$. Registros brasileiros de $1982^{3}$, baseados em diagnósticos histopatológicos, dão incidência para o câncer do ovário de 715 casos anuais, para uma população que na época era de 62.341.214 mulheres residentes no território nacional ${ }^{7}$. No Censo demográfico de 2000, o Instituto Brasileiro de Geografia e Estatística (IBGE) levantou 169.799.170 pessoas residentes, com um total de 86.223.155 mulheres residentes. Para o ano de 2005, estimou-se uma população de 184.184.074 com 93.513.055 mulheres ${ }^{7}$. Transgredindo os dados, uma vez que a incidência dessa neoplasia mantém-se estável no Brasil ${ }^{4}$, esperavam-se aproximadamente 1072 novos casos, para o ano de 2005. As tabelas 1 e 2 informam sobre valores absolutos e taxas de incidência dessa neoplasia em algumas cidades brasileiras, segundo os dados de Registros de Câncer em Base Populacional (INCA) ${ }^{8}$, conforme últimas atualizações realizadas nesses registros.

A taxa de sobrevida geral, em 5 anos, é de 50\%, com $95 \%$ de sobrevida para paciente com neoplasia restrita aos ovários (estadiamento I), 79\% naquelas com doença infiltrada em tecidos adjacentes aos ovários (estadiamento II) e de $28 \%$ naquelas com estádio clínico avançado (estadiamento IV) ${ }^{1}$. Na Escócia, a taxa global de sobrevida para pacientes diagnosticadas com câncer de ovário, entre 1991 a 1995, foi em um ano de 56,3\% e de $28,9 \%$, em cinco anos 9 . Em 2000, a taxa global de sobrevida, em cinco anos, para o carcinoma de ovário foi $39,1 \%{ }^{10}$. Em 2000, O Departamento de Ginecologia do Hospital AC Camargo obteve sobrevida global de $58,4 \%{ }^{11}$. A média de idade dessa neoplasia está entre 40 e 65 anos $^{12}$, sendo a sexta década de vida a idade mais prevalente ao diagnóstico ${ }^{4}$.

As neoplasias epiteliais malignas correspondem de 90 a 95\% das neoplasias malignas ovarianas. A Organização Mundial de Saúde (OMS) classifica os tumores epiteliais ovarianos em nove grupos (tabela 3) que podem ser sub-classificados, de acordo com o padrão de crescimento, em císticos, sólidos, papilíferos, entre outros; quanto à quantidade de estroma fibroso no tumor; e quanto ao comportamento biológico, conforme o grau de atipias e invasibilidade, em benignos, malignos e boderlines, sendo que os últimos possuem comportamento biológico intermediário ${ }^{13}$.

Por sua prevalência e seu desenvolvimento silencioso, 
Tabela 1. Valores absolutos de casos novos de câncer em cidades brasileiras, segundo última atualização disponível nos Registros de Câncer de Base Populacional, INCA

\begin{tabular}{l|c|c|c|c}
\hline \multicolumn{1}{c|}{ Cidade } & Ano & $\begin{array}{c}\text { Casos novos de câncer } \\
\text { na população geral }\end{array}$ & $\begin{array}{c}\text { Casos novos de câncer } \\
\text { no sexo feminino }\end{array}$ & $\begin{array}{c}\text { Casos novos } \\
\text { de câncer de ovário }\end{array}$ \\
\hline Campinas (SP) & 1995 & 1305 & 700 & 21 \\
Curitiba (PR) & 1998 & 3456 & 1922 & 66 \\
Belém (PA) & 1998 & 1324 & 827 & 32 \\
Fortaleza (CE) & 1999 & 4407 & 2664 & 117 \\
Manaus (AM) & 1999 & 1890 & 1088 & 34 \\
Belo Horizonte (MG) & 2000 & 11619 & 6232 & 124 \\
Goiânia (GO) & 2000 & 2160 & dado não disponível & 41 \\
Porto Alegre (RS) & 2000 & 5894 & 3049 & 114 \\
Palmas (TO) & 2001 & 183 & 116 & 4 \\
Recife (PE) & 2001 & 2973 & 642 & 28 \\
Cuiabá (MT) & 2002 & 1233 & 1950 & 66 \\
Salvador (BA) & 2002 & 3435 & & \\
\hline
\end{tabular}

Tabela 2. Taxas de incidência de câncer, nas áreas de coberturas e períodos assinalados, segundo localização de câncer primário, com intervalo de confiança de 95\%, conforme dados dos Registros de Câncer de Base Populacional, INCA

\begin{tabular}{c|c|c|c|c}
\hline Período & Área de cobertura & $\begin{array}{c}\text { No total de casos } \\
\text { novos de câncer no } \\
\text { sexo feminino }\end{array}$ & $\begin{array}{c}N^{\circ} \text { casos novos } \\
\text { de câncer de } \\
\text { ovário }\end{array}$ & $\begin{array}{c}\text { Taxa bruta de incidência de } \\
\text { casos novos de câncer de } \\
\text { ovário / 100.000 mulheres }\end{array}$ \\
\hline $1991-1995$ & Campinas (SP) & 3255 & 107 & 4,81 \\
$1995-1999$ & Fortaleza (CE) & 10144 & 400 & 7,46 \\
$1995-1999$ & Porto Alegre (RS) & 12337 & 447 & 12,96 \\
$1996-1998$ & Belém (PA) & 2264 & 63 & 3,45 \\
$1998-1998$ & Curitiba (PR) & 1922 & 66 & 8,21 \\
$1998-2002$ & Salvador (BA) & 7775 & 263 & 4,13 \\
$2000-2000$ & Belo Horizonte (MG) & 6232 & 124 & 10,50 \\
$2000-2001$ & Palmas (TO) & 181 & 4 & 2,78 \\
$2002-2002$ & Cuiabá (MT) & 2261 & 61 & 5,6 \\
\hline
\end{tabular}

torna-se importante discorrer sobre a patogênese da neoplasia maligna epitelial de ovário, a menos clara entre os tumores genitais femininos ${ }^{14}$, que se não detectada precocemente produz elevada mortalidade, apesar dos avanços no manejo da doença avançada 5 .

Esse estudo objetivou uma análise crítica de pesquisas previamente publicadas em periódicos indexados nas bases de dados Medline e Scielo, a respeito dos fatores de risco e patogênese da neoplasia epitelial ovariana, sendo selecionadas pesquisas caso-controle, randomizadas e revisões de literatura. Os unitermos utilizados foram "carcinomas de ovário", "fatores de risco" e "epidemiologia", sendo selecionados artigos a partir de 1968 até a data corrente, a fim de realizar uma revisão de literatura abrangente. No total, foram analisados 87 artigos indexados nas bases de dados relacionadas. Para outras informações epidemiológicas, foram acessados os bancos de dados do Ministério da Saúde e da Organização Mundial da Saúde (OMS).

\section{TEORIAS CARCINOGÊNICAS EM TECIDO EPITELIAL OVARIANO}

Em 1971, Fathalla ${ }^{15}$ propôs que podem ocorrer alteraçôes genéticas na superfície epitelial do ovário em resposta a uma incessante ovulação, por microtraumas e injúrias de repetição. Ele teorizou ainda a possibilidade de ativação de mitoses celulares sucessivas, que podem desencadear mutagênese e, por conseguinte, carcinogênese. Em 1978, Zajicak ${ }^{16}$ sugeriu que a inclusão cística epitelial na superfície do ovário pode ser fonte de neoplasia, uma vez que há uma relação modesta entre o número de cistos germinativos de inclusão e idade ovulatória, fornecendo evidências que estes podem ser causados durante período ovulatório ou pelo menos associados a ele ${ }^{17}$. Casagrande ${ }^{18}$ (1979) 
Tabela 3. Classificação histológica dos tumores epiteliais de superfície ovariana, OMS

\begin{tabular}{|c|c|c|}
\hline & Classificação histológica & Sub-classificação histológica, exemplos \\
\hline I & Serosos & $\begin{array}{l}\text { Cistoadenoma seroso (benigno); tumor boderline (de } \\
\text { malignidade limítrofe); cistoadenocarcinoma seroso } \\
\text { (maligno); adenofibroma seroso; cistoadenofibroma } \\
\text { seroso. }\end{array}$ \\
\hline II & Mucinosos & $\begin{array}{l}\text { Cistoadenoma mucinoso (benigno); tumor boderline (de } \\
\text { malignidade limítrofe); cistoadenocarcinoma mucinoso } \\
\text { (maligno); adenofibroma mucinoso. }\end{array}$ \\
\hline III & Endometrióides & Benignos; de malignidade limítrofe; malignos. \\
\hline IV & Células claras & Benignos; de malignidade limítrofe; malignos. \\
\hline V & Células transicionais & $\begin{array}{l}\text { Tumor de Brenner; tumor de Brenner (malignidade } \\
\text { limítrofe); tumor de Brenner maligno; carcinoma de } \\
\text { células de transição (tipo não-Brenner). }\end{array}$ \\
\hline VI & Células escamosas & $\begin{array}{l}\text { Benignos; intermediários; carcinoma de células } \\
\text { escamosas. }\end{array}$ \\
\hline VII & Tumores epiteliais mistos & Tumor mülleriano; adenossarcoma. \\
\hline VII & $\begin{array}{l}\text { Tumores indiferenciados e } \\
\text { não-classificados }\end{array}$ & Tumores malignos de estrutura epitelial. \\
\hline IX & Outros & Carcinoma adenóide-cístico; carcinoma basalóide. \\
\hline
\end{tabular}

relacionou o risco de desenvolver carcinoma de ovário e o número de ovulações, entre menarca e menopausa, com o risco de desenvolvimento dessa neoplasia. Conforme esta proposta, os períodos de anovulação são considerados fatores de proteção. Deve-se lembrar ainda que o tecido ovariano é responsivo a gonadotrofinas e, portanto, um estado hipergonadotrófico é fator contribuinte para desenvolvimento de carcinoma de ovário $^{19,20}$. Cramer e Welch ${ }^{21}$, em 1983, sintetizaram essas teorias em um modelo no qual inclusōes císticas epiteliais recebem estimulações de altas taxas de gonadotrofinas e seus efeitos diretos e indiretos na esteroidogênese induzem carcinoma.

Segundo essas teorias, quanto maior for o período de permanência em anovulação em uma mulher, maior será a proteção do epitélio ovariano a processos carcinogênicos. Os períodos de anovulação correspondem a situações como gravidez, uso de anticoncepcionais hormonais orais (ACHO), amamentação, pequeno período menstrual na vida reprodutiva, como menarca tardia e menopausa precoce. Vários autores ${ }^{22,23,24}$ concluíram que para períodos de anovulação, independente da idade, superior ou inferior a 55 anos, os principais contribuintes para que o risco de carcinoma epitelial ovariano diminua são gravidez a termo, uso de $\mathrm{ACHO}$ e amamentação. Embora não tenham encontrado grande impacto sobre esse risco, em relação à menarca tardia e menopausa precoce, observaram que a ação protetora da anovulação em tecido epitelial ovariano é mais notória por períodos consecutivos mais prolongados do que em ciclos intermitentes durante a vida reprodutiva ${ }^{23,25}$. Outros autores observaram que o tempo de ovulação está envolvido na patogênese carcinogênica em mulheres em pré-menopausa, mas não naqueles carcinomas ovarianos encontrados na pós-menopausa ${ }^{24}$. Observaram também que carcinomas ovarianos do tipo não-mucinoso estão mais relacionados com período menstrual e ciclos ovulatórios do que com os carcinomas mucinosos ${ }^{26}$. No entanto, um estudo maior, também caso-controle, evidenciou que o uso de $\mathrm{ACHO}$, paridade, amamentação, ligação tubária, histerectomia, idade na menarca e menopausa, e história familiar positiva para carcinoma ovariano não determinaram diferenças nos subtipos histopatológicos nos carcinomas malignos diagnosticados ${ }^{27}$.

Em relação à teoria das gonadotrofinas, ela foi evidenciada em experimentos animais ${ }^{28,29}$, onde foi constatado que o tratamento com agonista do hormônio liberador de gonadotrofinas $(\mathrm{GnRH})$ reduz a incidência de carcinoma de ovário ou inibe o crescimento desse quando já presente. Henderson et al..$^{30}$ sugeriram que estimulação persistente do ovário por gonadotrofinas pode ter efeito direto ou indireto na carcinogênese de 
ovário por aumentar os níveis de estrógenos. Contudo, é necessário ter ciência que alguns autores não encontraram relação direta ou indireta entre carcinomas ovarianos e gonadotrofinas ${ }^{31,32,33}$.

\section{FERTILIDADE E GESTAÇÃO}

Sabe-se que concomitante à gestação ocorrem anovulação e supressão pituitária e, logo, há diminuição no risco carcinogênico sobre epitélio ovariano ${ }^{34,35}$. Assim, teoricamente, cada gestação adicional a termo está associada com diminuição de risco de carcinoma epitelial ovariano invasivo ${ }^{22}$ e carcinoma de baixo potencial maligno ou boderlines ${ }^{36}$. A primeira gestação a termo reduz em aproximadamente $40 \%$ o risco desse tipo de câncer, no entanto os dados sugerem que cada gestação adicional à primeira confere uma diminuição de $14 \%$ no risco para carcinoma invasivo epitelial de ovário $^{23}$. Alguns estudos consideram essa porcentagem entre $15-20 \%{ }^{37}$. Percebe-se, então, que a magnitude protetora da primeira gestação é maior do que as demais $^{38,39}$. Gestações não levadas a termo não protegem contra o carcinoma como aquelas que são levadas. Contudo, vários autores as consideram como fator protetor $^{22,40}$. Um estudo norte-americano demonstrou que gestações não levadas a termo têm efeito protetor apenas entre pacientes que já tiveram o primeiro filho. Eles não encontraram associação estatística entre o carcinoma de ovário e nulíparas que apresentaram gravidez incompleta espontânea ou induzida ${ }^{41}$.

O efeito da idade durante a primeira gestação sobre o risco de carcinoma de ovário epitelial desapareceu quando corrigido em relação ao número de gestaçôes ${ }^{42}$. No entanto, quanto mais tarde a mulher gestar, maior é a magnitude protetora dessa gravide $z^{40}$.

Em relação à infertilidade, vários estudos têm indicado que ela é um fator independente para o risco de câncer de ovário, separadamente do efeito de nuliparidade ${ }^{43-47}$. Joly et al. ${ }^{44}$ (1974) concluíram que mulheres que desenvolveram câncer de ovário tinham um estado gonadal que facilitava tanto o desenvolvimento da neoplasia quanto a baixa fertilidade. Nasca et al. ${ }^{45}$ (1984) definiram que os fatores determinantes da infertilidade são importantes para correlacionar baixa paridade, gravidez e risco aumentado de câncer de ovário. Booth et al. ${ }^{46}$ (1989) concluíram que mulheres que não conceberam após 10 anos de tentativa, sem utilização de nenhum método contraceptivo, tinham 6,5 vezes maior risco de desenvolver câncer comparadas a nulíparas que estavam tentando gestar há menos de 3 meses, sem utilização de nenhum método contraceptivo. Whittemore et al. ${ }^{47}$ (1989) determinaram que o risco de neoplasia de ovário para mulheres com mais de dez anos de tentativas desprotegidas era 1,8 vezes maior do que em mulheres reportando apenas alguns anos de tentativas. Em resumo, esses estudos sugerem que fatores que culminam em alterações de fertilidade exercem função importante no desenvolvimento de câncer de ovário, tipo epitelial. Shoham ${ }^{48}$ concluiu que um fator, provavelmente de origem gonadal, é responsável por carcinogênese ovariana e diminuição de fertilidade, em pelo menos uma parcela das pacientes inférteis. Mosgaard et al. ${ }^{49}$ afirmaram que o risco para desenvolver câncer de ovário, em nulíparas, dobra. E finalmente, Risch et al. ${ }^{50}$ observaram que mulheres nulíparas, voluntariamente, constituem grupo de risco alto, no qual a infertilidade, mesmo sendo voluntária, é fator predisponente para carcinoma de ovário.

Quanto ao uso de drogas indutoras de ovulação, que agem elevando os níveis de FSH (hormônio folículo estimulante), os estudos confirmam e nos orientam sobre seu envolvimento na patogênese de tumores boderlines de ovário, quando administradas por tempo superior a 12 meses $^{51}$, sendo esses considerados pela OMS primários às neoplasias epiteliais.

Fatores que dificultam a avaliação da patogênese da neoplasia ovariana epitelial em relação à paridade são as várias causas ligadas à infertilidade e à dificuldade em determinar quando uma paciente é nulípara infértil ou nulípara voluntária.

\section{AmAmentação}

É capaz de inibir parcialmente a ovulação ${ }^{52,53}$ pela refratariedade aos níveis de FSH e de hormônio luteinizante (LH) elevados ${ }^{54}$ e, assim, teoricamente se tem diminuição de risco carcinogênico em epitélio ovariano. Concomitantemente, como há elevação dos níveis de FSH e $\mathrm{LH}^{55}$ teoricamente também há favorecimento da ação estrogênica em tecido ovariano ${ }^{55}$ e logo, aumento do risco. Alguns estudos ${ }^{22,23}$ revelam que a refratariedade ao FSH tem efeito protetor sobre o risco de carcinoma de ovário epitelial, diminuindo-o, se estiver presente nos primeiros meses, ao menos, pósnascimento do beb $\hat{e}^{54}$.

Torna-se difícil avaliar o fator protetor da amamentação isoladamente, uma vez que está relacionada à gestação. Assim, muitos estudos são favoráveis ao seu papel protetor ${ }^{56}$, independentemente da idade da paciente, 
pré ou pós-menopausas ${ }^{24}$, enquanto alguns afirmam seu efeito protetor apenas antes da menopausa ${ }^{57}$. Alguns autores revelam que a amamentação somente tem efeito protetor em mulheres cujo índice de massa corpórea (IMC) é inferior a $35^{22}$. Outros encontraram que o tempo de lactação, apenas acima de um ano, tem esse efeito ${ }^{58}$; ao passo que há relatos de que pode haver proteção apenas para carcinomas ovarianos não-mucinosos, através da amamentação ${ }^{26}$. Há dados que demonstram não haver associação, nem protetora, nem de risco, entre lactação e câncer de ovário ${ }^{40}$.

\section{USO DE ANTICONCEPCIONAIS HORMONAIS ORAIS E HORMÔNIOS ESTERÓIDES EXÓGENOS}

$\mathrm{O}$ estrogênio das pílulas anticoncepcionais é capaz de suprimir a ovulação e reduzir a secreção de gonadotrofinas pela pituitária ${ }^{59}$ e, assim, pode-se diminuir o risco de câncer de ovário, tipo epitelial. Vários estudos confirmam esses dados epidemiologicamente: há diminuição do risco de carcinoma de ovário com o uso prolongado de anticoncepcionais hormonais orais ${ }^{22,23,60-62}$, em até $50 \%$, para um uso de cinco $\operatorname{anos}^{37}$, sendo essa relação mais pronunciada em nulíparas ${ }^{63}$. Os estrógenos conjugados suprimem os níveis de hormônios da pituitária somente em mulheres menopausadas, embora dados a respeito de terapia hormonal nessas mulheres não mostraram diminuição no risco desse carcinoma ${ }^{59,60,64-67,68}$. Segundo Whittemore et $\mathrm{al}^{23}$, em poucas pacientes usuárias de ACHO, os níveis de gonadotrofinas encontram-se elevados para estarem relacionados com a carcinogênese.

Riman et al. ${ }^{37}$ demonstraram aumento no risco de câncer de ovário, em até 50\%, quando comparadas mulheres usuárias e não usuárias de terapia de reposição hormonal (TRH), sendo o aumento no risco proporcional ao tempo de uso. Outros autores observaram que a relação entre uso de TRH e carcinoma de ovário é mais forte em mulheres não-histerectomizadas ou nãolaqueadas ${ }^{69}$. Ainda não se sabe porque a TRH pode diminuir os níveis de $\mathrm{FSH}$ e $\mathrm{LH}$, mas não diminui o risco do carcinoma de ovário. Acredita-se que, durante o período da menopausa, há vários fatores presentes na superfície do epitélio ovariano, como fatores angiogênicos, genes moduladores e de crescimento e moléculas de adesão, e que estes estão relacionados a esse comportamento, nessa faixa populacional ${ }^{68}$.

\section{IDADE NA MENARCA E NA MENOPAUSA}

Segundo as mesmas teorias, mulheres que começam a menstruar precocemente e têm sua menopausa tardiamente são mais propensas ao carcinoma de ovário. Esse risco aumenta naquelas cuja menarca precede aos dez anos de $\operatorname{idade}^{23}$. Na rotina, observa-se que a menopausa precoce interfere pouco sobre o risco da carcinogênese, uma vez que durante o período de climatério ocorrem ciclos ovulatórios esporádicos, diminuindo o impacto sobre o fator anovulação e, assim, observa-se que idade avançada na menopausa é fracamente associada ao aumento do risco de câncer de ovário tipo epitelial ${ }^{37}$. Alguns estudos colocam-se a favor do decréscimo, embora pequeno e não significativo, sobre o risco de carcinoma ovariano epitelial e outros não se colocam totalmente a favor desse resultado ${ }^{23}$. Para analisar esses dados, devemos nos recordar que níveis continuamente elevados de gonadotrofinas, em menopausadas tardiamente, deveriam aumentar o risco de câncer nessa fração populacional ${ }^{70}$.

\section{CiRURGIAS PÉLVICAS}

Ligação tubária e histerectomia, com conservação de ovários, podem comprometer o suplemento sanguíneo ovariano $^{23}$. Com isso, as seqüelas cirúrgicas desses procedimentos, inclusive as circulatórias, agem diminuindo a ação hormonal e, assim, reduzem esse risco ${ }^{71}$. Essa proteção tende a ser maior em histerectomizadas em idade fértil do que em menopausadas. No entanto, com o efeito de feedback, há aumento de produção de gonadotrofinas e teoricamente o risco poderia estar aumentado ${ }^{23}$.

$\mathrm{O}$ risco de neoplasia de ovário, em mulheres que realizam ligação tubária ou histerectomia, diminuiu em estudos prospectivos e retrospectivos realizados ${ }^{71,72,73}$. Riman et al. ${ }^{37}$ informaram que o risco de câncer de ovário pode diminuir em torno de $80 \%$ em mulheres histerectomizadas ou laqueadas.

\section{FATORES GenÉticos}

O tipo histológico epitelial de neoplasia maligna ovariana pode estar relacionado à história familiar de carcinoma da mama, ovário ou ambos e nessas pacientes pode incidir em idade mais precoce do que em população geral ${ }^{5}$. Essa síndrome familiar está relacionada à mutação em dois genes, o BRCA-1 e BRCA-2, localizados respectivamente nos cromossomos $17 \mathrm{q}$ e $13 \mathrm{p}$, envolvidos no processo de reparo de $\mathrm{DNA}^{5}$. Outra síndrome associada ao aumento de risco de câncer de ovário hereditário é a síndrome de Lynch II, com mutação nos genes MSH2 ou MLH1, também relacionada a tumores de cólon não-poliposos e endométrio. Pesquisas informam que história positiva de câncer de ovário, em dois ou mais parentes de 
primeiro grau, aumenta significativamente o risco de câncer de ovário ${ }^{60}$.

Parazzini et al. ${ }^{74}$, em estudo caso-controle italiano, conduzido de 1983 a 1989, com 755 casos de carcinoma epitelial de ovário e 2.023 controles, confirmaram que a história familiar aumenta o risco da doença, mas a porcentagem de casos de câncer de ovário explicados por história familiar é pequena: menos de $1 \%$ dos casos observados no estudo demonstraram serem atribuídos ao risco familiar. A literatura discorre que aproximadamente $5 \%{ }^{6}$ a $10 \%{ }^{61}$ dos casos de carcinoma epitelial ovariano invasivo são hereditários, com presença da mutação em BRCA-1 e BRCA-2.

Um estudo ${ }^{75}$ comparou os efeitos do uso de ACHO, paridade, amamentação e ligação tubária em 242 mulheres judias com diagnóstico de carcinoma ovariano invasivo, divididas em grupos conforme as mutaçōes encontradas: 185 delAG e 5382insC em BRCA-1, e 6174delT em BRCA-2. Eles não encontraram diferenças entre os grupos quanto ao uso de $\mathrm{ACHO}$, paridade, e amamentação. No entanto, o grupo BRCA-1 apresentou maiores índices de ligação tubária do que o grupo BRCA-2.

Em 2001, foi publicado um estudo ${ }^{60}$, com 751 controles e 840 casos, que acessava os efeitos da paridade e do uso de ACHO no risco de carcinoma de ovário entre mulheres judias, grupo étnico com alta incidência de mutação de genes BRCA-1 e BRCA-2, em Israel, para determinar se o uso de $\mathrm{ACHO}$ e multiparidade abaixa o risco dessa patologia nessas pacientes. Apenas $1,7 \%$ dos controles apresentava essa mutação e entre os casos, $29 \%$. No grupo não-portador da mutação, cada gestação/nascimento conferia uma diminuição de $12 \%$ no risco dessa neoplasia. No grupo portador, cada gestação/nascimento conferia um decréscimo de $6 \%$ no risco. $\mathrm{O}$ uso prolongado de $\mathrm{ACHO}$ não determinou diminuição do risco dessa patologia, embora se tenha encontrado diminuição de risco nas pacientes mais velhas, provavelmente porque essas usavam pílulas com maior dosagem hormonal. Narod et al. ${ }^{61}$, em seu estudo caso-controle, com 207 casos de neoplasia ovariana hereditária e 161 controles, grupo composto por irmãs das portadoras da mutação, concluíram que o uso de ACHO pode diminuir o risco desse carcinoma, nesse grupo específico.

Esses dados nos indicam que o uso de $\mathrm{ACHO}$, como prevenção dessa patologia, em mulheres portadoras dessa mutação, talvez seja prematuro, mas até o momento acredita-se que se trata de fator protetor.

\section{OUTROS FATORES}

Rish et al. ${ }^{76}$ consideraram que o risco de câncer pode aumentar, se houver excesso de estimulação androgênica, em células epiteliais ovarianas, e pode diminuir quando se relaciona com estimulação progestagênica. Eles fundamentaram sua hipótese no estudo de Helzhauer et al. ${ }^{77}$, no qual foram evidenciados altos níveis de androstenediona e dihidroepiandrosterona em câncer de ovário. Esse estudo nos alerta sobre a relação entre risco elevado de carcinoma de ovário e síndrome dos ovários policísticos. Essa síndrome também está relacionada com menores períodos de ovulação na vida reprodutiva dessas pacientes e, assim, poderia também estar relacionada à diminuição de risco de carcinoma invasivo de ovário. No entanto, aqueles fatores já mencionados como gravidez, uso de ACHO e amamentação são mais protetores do que a síndrome dos ovários policísticos, uma vez que nestas pacientes os períodos anovulatórios tendem a não serem consecutivos. Relacionada a essa síndrome, está a obesidade e, assim, também é intrínseca a proteção a carcinomas ovarianos, uma vez que anovulação também é mais freqüente nessas mulheres ${ }^{66}$. A hiperinsulinemia também está associada à hiperprodução de androgênios ovarianos e a acantose nigricans é freqüentemente observada em pessoas com alto grau de resistência à insulina. Assim, essas situações podem estar envolvidas com períodos anovulatórios e fator de proteção ao carcinoma de ovário ${ }^{78}$, embora alguns estudos tenham fundamentado que hiperandrogenismo eleva o risco de carcinoma de ovário.

Paulson $^{79}$ sugeriu que a endometriose, como fator marcador da presença de menstruação retrógrada, determinando um aumento de fluido intraperitoneal, é capaz de auxiliar no desenvolvimento de câncer de ovário, pelo constante fator irritativo e conseqüente reação inflamatória. Essa teoria foi aceita quando se concluiu que o risco de neoplasia de ovário em mulheres que realizam ligação tubária ou histerectomia diminuiu ${ }^{71,72,80,81}$. Modugno et al..$^{82}$ investigaram o risco relativo para câncer de ovário, em portadoras desse carcinoma, considerando o uso de $\mathrm{ACHO}$, paridade, histerectomia e ligação tubária, em dois grupos: controle, não portador de endometriose, e grupo portador de endometriose. Eles encontraram similar redução no risco relativo considerando as variáveis analisadas, em ambos os grupos. Porém, mulheres com endometriose tiveram aumento de risco para carcinoma ovariano. Para esse grupo particular, portador de endometriose, eles descobriram que o uso de $\mathrm{ACHO}$ por mais de 10 anos 
diminuiu substancialmente o risco desse carcinoma.

A respeito dos efeitos ambientais na carcinogênese de tecido ovariano, a exposição do epitélio ovariano ao talco pode estar envolvida, uma vez que este pode estar contaminado com asbesto e, assim, relacionar-se com aumento de mortalidade, como ocorre em outros cânceres $^{83}$. Dessa maneira, alguns estudos sobre o risco de desenvolvimento de carcinoma e uso de diafragmas com talco foram desenvolvidos, sendo que alguns resultados sugeriram uma relação verdadeira entre esses fatores $^{84,85}$ e outros, uma relação falsa ${ }^{46,86-88}$.

Com base em modelos animais e estudos ecológicos, foi proposto que o consumo diário de açúcar e lactose aumenta o risco de câncer de ovário. O consumo diário de produtos derivados do leite foi assim considerado, porque o metabolismo de carboidratos, galactose $\mathrm{e}$ lactose podem ser tóxicos a oócitos ${ }^{89}$. Um estudo ${ }^{90}$ analisou, em metanálise, oito trabalhos caso-controle, não evidenciando associação entre ingestão diária de lactose no risco dessa neoplasia. No entanto, o resultado desse trabalho quanto à análise de três estudos de coorte encontrou associação significativamente positiva entre ingestão de lactose e neoplasia de ovário, mesmo sendo uma ingesta pequena, de um copo de leite ao dia. Outro trabalho de metanálise ${ }^{89}$, também, avaliou a influência da lactose no risco de neoplasia de ovário, em 20 estudos caso-controle e dois estudos de coorte. A análise estatística não encontrou qualquer associação entre ingesta desses produtos e risco aumentado, significativo, de carcinoma de ovário, embora tenha se evidenciado um aumento pequeno desse.

Baseando-se na evidência de que inflamação crônica pode influenciar na carcinogênese epitelial ovariana, vários estudos passaram a analisar sua interferência, partindo da premissa de que anti-inflamatórios nãohormonais (Aines), como aspirina e acetominofeno, alteram a resposta inflamatória no organismo ${ }^{91-94}$. O uso de três ou mais vezes por semana de aspirina por período maior ou igual a 6 meses pode reduzir em $40 \%$ o risco dessa neoplasia ${ }^{91}$. Há uma relação inversa significativa entre o uso de paracetamol e risco de câncer de ovário ${ }^{92}$. Esses autores ${ }^{92}$ encontraram também uma associação preventiva, mas não suficientemente significativa, entre uso de aspirina e a neoplasia assinalada. No entanto, não encontraram evidência de proteção em relação à utilização de ibuprofeno. Eles fundamentaram seus achados em um estudo em roedores, no qual se evidenciou que altas doses de paracetamol produzem atrofia ovariana e uterina e diminuição de formação de cistos ovarianos. Em um estudo ${ }^{93}$ de seguimento de 16 anos, com 333 casos de neoplasia epitelial invasiva de ovário, não se observou associação entre duração ou dose de uso de aspirina e o carcinoma. No entanto, observaram uma redução modesta, não-significativa, no risco da neoplasia em relação ao uso de Aines, sem evidência de melhora da prevenção quanto à dosedependência. Um estudo experimental ${ }^{94}$ com células in vitro não foi conclusivo para determinar os efeitos dos Aines como quimioprevenção do câncer ovariano. Dessa forma, a dose ideal da aspirina ainda está em fase de estudo, concluindo-se, portanto, que ainda é muito cedo para afirmar o real benefício de seu uso como prevenção do câncer de ovário.

\section{CONCLUSÃO}

Concisamente, essas informações nos orientam sobre os fatores fisiopatológicos envolvidos na carcinogênese de tecido ovariano. Podemos citar como importantes dados a serem considerados em estudos melhores conduzidos: idade da paciente, paridade, amamentação, fatores de infertilidade, utilização de drogas indutoras de ovulação, qual espécime e por quanto tempo, etnia, administração de anticoncepcionais hormonais orais, fatores ambientais, fatores cirúrgicos e história pregressa de patologias, como a endometriose.

Para a pesquisa da neoplasia maligna epitelial de ovário em mulheres, o perfil epidemiológico associado à identificação de fatores de risco continua sendo o melhor recurso de que dispomos para uma detecção precoce dessa patologia, uma vez que até o momento não há programas de controle para os fatores de risco citados e o rastreamento, através de marcadores séricos (CA-125) e ultra-sonografias (transvaginal e pélvica), é preconizado para mulheres com antecedentes familiares de câncer ovariano genético.

\section{REFERÊNCIAS BIBLIOGRÁFICAS}

1. Smith H. Clinical Management of Ovarian Cancer [book review]. N Engl J Med. 2001; 345(2):152-3.

2. Instituto Nacional de Câncer; Ministério da Saúde. Estimativa da incidência de câncer no Brasil: 1999. Rio de Janeiro: INCA; 1999.

3. Brumini R, organizador. Câncer no Brasil: dados histopatológicos: 1976-1980. Rio de Janeiro: Ministério da Saúde; 1982.

4. Pascalicchio JC, Fristachi CE, Castanho PROL, Kue CM, Piato S, Baracat FF. Epidemiologia do câncer de ovário no Brasil. Rev Soc Bras Canc. 2000;11: 46-53.

5. Cannistra SA. Cancer of the ovary. N Engl J Med. 2004;351: 2519-29. 
6. Benedet H, Bender H, Jones III, Ngan HYS, Pecorelli S. Staging classifications and clinical practice guidelines of gynaecologic cancers. Int J Gynecol Obstet. 2000;70: 207-12.

7. População residente de 1980 a 2005: Censos 1980, 1991 e 2000, Contagem 1996 e projeções intercensitárias [base de dados na Internet]. Brasília: Sistema Único de Saúde; Ministério da Saúde; 2005 - [citado em 2005 Set 8]. Disponível em: http://tabnet.datasus.gov.br/cgi/ deftohtm.exe?ibge/cnv/popuf.def

8. Instituto Nacional de Câncer; Ministério da Saúde [homepage na Internet]. Rio de Janeiro: INCA; c19962006 [citado em 23 Out 2005]. Vigilância epidemiológica: sistema de câncer de base populacional; [cerca de 2 telas]. Disponível em: http://www.inca.gov.br/cgi/sisbasepop.asp

9. Scottish Cancer Intelligence Unit. Trends in cancer survival in Scotland 1971-1995 [monografia na Internet]. Edinburgh: Information and Statistics Division; 2000 [cited 2005 Jul 9]. Available from: http://www.isdscotland.org/ isd/files/trends_1971-95.pdf.

10. Heintz APM, Odicino F, Maisonneuve P, Beller U, Benedet JL, Creasman WT, et al. Carcinoma of the ovary. In: Pettersson F, editor. FIGO Annual Report: Proceedings of the 24th FIGO Annual Report on the Results of Treatment in Gynecological Cancer; 2002; Basingstoke, United Kingdom. [Oxford: Isis Medical Media; 2001].

11. Abrão FS, Jales AFC, Oyagawa L, Abrão MS, Novik PR, Breitbarg RC, et al. Rotina do Departamento de Ginecologia do Hospital A.C. Camargo. Acta Oncol. 2000;20(4):158.

12. Crum CP. Aparelho Genital Feminino. In: Cotran RS, Kumar V, Collins T, editores. Robbins: patologia estrutural e funcional. 6a. ed. Rio de Janeiro: Guanabara Koogan; 2000. p. 956-7.

13. World Health Organization of Tumours. Pathology \& Genetics. tumours of the breast and female genital organs. Lyon: IARC Press; 2003.

14. Baker TR, Piver MS. Etiology, biology, and epidemiology of ovarian cancer. Semin Surg Oncol. 1994;10:224.

15. Fathalla MF. Incessant ovulation: a factor in ovarian neoplasia? [letter]. Lancet. 1971;2: 163.

16. Zajicek J. Prevention of ovarian cystomas by inhibition of ovulation: a new concept. J Reprod Med. 1978;2:114.

17. Heller DS, Murphy P, Westhoff C. Are germinal inclusion cysts markers of ovulation? Gynecol Oncol. 2005;96(2):496-9.

18. Casagrande JT, Pike ML, Ross RK, Louis EW, Roy S, Henderson BE. Incessant ovulation and ovarian cancer. Lancet. 1979; 2:170-2.

19. Daly MB. The epidemiology of ovarian cancer. Hematol Oncol Clin North Am. 1992; 6:729.

20. Stadel BV. The etiology and prevention of ovarian cancer. Am J Obstet Gynecol. 1975; 123:772-4.

21. Cramer DW, Welch WR. Determinants of ovarian cancer risk II: inferences regarding pathogenesis. J Natl Cancer Inst. 1983;71:717-21.

22. Whittemore AS, Harris R, Itnyre J. Characteristics relating to ovarian cancer risk: collaborative analysis of 12 US casecontrol studies. II. Invasive epithelial ovarian cancer in white women. Am J Epidemiol. 1992;136:1184-203.

23. Whittemore AS, Harris R, Itnyre J. The Pathogenesis of Epithelial Ovarian Cancer. Am J Epidemiol. 1992; 136(10):1213-20.

24. Tung KH, Wilkens LR, Wu AH, McDuffie K, Nomura AM, Kolonel LN, et al. Effect of anovulation factors on pre- and postmenopausal ovarian cancer risk: revisiting the incessant ovulation hypothesis. Am J Epidemiol. 2005;61(4):321-9.

25. Metcalf MG. Incidence of ovulation from the menarche to the menopause: observations of $622 \mathrm{New}$ Zealand women. N Z Med J. 1983;96:645-8.

26. Tung KH, Goodman MT, Wu AH, McDuffie K, Wilkens LR, Kolonel LN, et al. Reproductive factors and epithelial ovarian cancer risk by histologic type: a multiethnic casecontrol study. Am J Epidemiol. 2003;158(7):629-38.

27. Modugno F, Ness RB, Wheeler JE. Reproductive risk factors for epithelial ovarian cancer according to histologic type and invasiveness. Ann Epidemiol. 2001;11(8):568-74.

28. Lux-Lantos VA, Thyssen SM, Chamson A, Libertun C. Effect of a gonadotrofin releasing hormone analog on an experimental ovarian tumor: direct and indirect actions. Life Sci. 1995;57:291-300.

29. Bllaakaer J, Baeksted M, Micic S, Albrectsen P, Rygaard J, Bock J. Gonadotrofin releasing hormone agonist suppression of ovarian tumorigenesis in mice of the $\mathrm{Wx} / \mathrm{Wv}$ genotype. Biol Reprod. 1995;53:775-9.

30. Henderson BE, Ross R, Bernstein L. Estrogens as a cause of human cancer: the Richard and Hinda Rosentahl Foundation award lecture. Cancer Res. 1998;48:246-53.

31. Graves PE, Surwit EA, Davis JR, Stouffer RL. Adenylate cyclase in human ovarian canceres: sensitivity to gonadotropins and non-hormonal activators. Am J Obstet Gynecol. 1985;153:877-82.

32. Nakano R, Kitamaya S, Yamamoto M, Shirma K, Ooshima A. Localization of gonadotropin binding sites in human neoplasms. Am J Obstet Gynecol. 1989;161:905-10.

33. Akhmedkhanov A, Toniolo P, Zeleniuch-Jacquotte A, Pettersson KS, Huhtaniemi IT. Luteinizing hormone, its beta-subunit variant, and epithelial ovarian cancer: the gonadotropin hypothesis revisited. Am J Epidemiol. 2001;154(1):43-9.

34. Parlow AF, Daane TA, Dignam WJ. On the concentration of radioimmunoassayable $\mathrm{FSH}$ circulating in blood throughout human pregnancy. J Clin Endocrinol Metab. 1970; 31:213-4.

35. Jeppsson S, Rannevik G, Thorell JI. Pituitary gonadotrophin secretion durting the first weeks of 
pregnancy. Acta Endocrinol. 1977;85:177-88.

36. Harris R, Whittemore AS, Itnyre J. Characteristics relating to ovarian cancer risk: collaborative analysis of 12 US casecontrol studies. III Epithelial tumors of low malignant potential in white women. Am J Epidemiol. 1992;136:1204-11.

37. Riman T, Nilsson S, Persson IR. Review of epidemiological evidence for reproductive and hormonal factors in relation to the risk of epithelial ovarian malignancies. Acta Obstet Gynecol Scand. 2004;83(9):783-95.

38. Adami HO, Hsieh CC, Lambe M, Trichopoulos D, Leon D, Persson I, et al. Parity, age at first childbirth, and risk of ovarian cancer. Lancet. 1994;344:1250-4.

39. Albrektsen G, Heuch I, Kvale G. Reproductive factors and incidence of epithelial ovarian cancer: a Norwegian prospective study. Cancer Causes Control. 1996;7:421-7.

40. Riman T, Dickman PW, Nilsson S, Correia N, Nordlinder $\mathrm{H}$, Magnusson CM, et al. Risk factors for invasive epithelial ovarian cancer: results from a Swedish case-control study. Am J Epidemiol. 2002;156(4):363-73.

41. Gierach GL, Modugno F, Ness RB. Relations of gestational length and timing and type of incomplete pregnancy to ovarian cancer risk. Am J Epidemiol. 2005;161(5):452-61.

42. Hartge P, Schiffman MH, Hoover R, McGowan L, Lesher L, Norris HJ. A case-control study of epithelial ovarian cancer. Am J Obstet Gynecol. 1989;161:10-6.

43. Harlow BL, Weiss NS, Roth GJ, Chu J, Daling JR. Casecontrol study of borderline tumors: reproductive history and exposure to exogenous female hormones. Cancer Res. 1988; 48: 5849-52.

44. Joly DJ, Lilienfeld AM, Diamond EL, Bross IDJ. An epidemiologic study of the relationship of reproductive experience to cancer of the ovary. Am J Epidemiol. 1984; 99:190-209.

45. Nasca PC, Greenwald P, Chorost S, Richert R, Caputo T. An epidemiologic case-control study of ovarian cancer and reproductive factors. Am J Epidemiol. 1984;119:705-13.

46. Booth M, Beral V, Smith P. Risk factors for ovarian cancer: a case control study. Br J Cancer. 1989;60:592-8.

47. Whittemore AS, Wu ML, Paffenbarger RS, Sarles DL, Kampert JB, Grosser S, et al. Ephitelial ovarian cancer and the ability to conceive. Cancer Res. 1989;49:4047-52.

48. Shoham Z. Epidemiology, etiology, and fertility drugs in ovarian epithelial carcinoma: where are we today? Fertil Steril. 1994;62:433-48.

49. Mosgaard BJ, Lidegaard O, Andersen AN. The impact of parity, infertility and treatment with fertility drugs on the risk of ovarian cancer. Acta Obstet Scand. 1997;76:89-95.

50. Risch HA, Loraine DM, Howe GR. Parity, contraception, infertility, and the risk of epithelial ovarian cancer. Am J Epidemiol. 1994;140(7):585-97.

51. Rossing MA, Daling JR, Weiss NS, Moor DE, Self SG. Ovarian tumors in a cohort of infertile women. N Engl J
Med. 1994;331:771-6.

52. Perez A, Vela P, Masnick GS, Potter RG. First ovulation after childbirth: the effect of breast-feeding. Am J Obstet Gynecol. 1972;114:1041-7.

53. Gray R, Eslami S, Campbell O. Return of fertility during lactation monitored by urinary steroid assays [abstract]. Am J Epidemiol. 1987;126:761.

54. Reyes FI, Winger JSD, Faiman C. Pituitary-ovarian relationships during the puerperium. Am J Obstet Gynecol. 1972;114:589-94.

55. Zarate A, Canales E. The reproductive age: the human ovary during pregnancy and puerperium. In: Serra GB, editors. The ovary. New York: Raven Press; 1983. p. 257-71.

56. Chiaffarino F, Pelucchi C, Negri E, Parazzini F, Franceschi $\mathrm{S}$, Talamini R, et al. Breastfeeding and the risk of epithelial ovarian cancer in an Italian population. Gynecol Oncol. 2005;98(2):304-8.

57. Siskind V, Green A, Bain C, Purdie D. Breastfeeding, menopause, and epithelial ovarian cancer. Epidemiology. 1997;8(2):188-91.

58. Yen ML, Yen BL, Bai CH, Lin RS. Risk factors for ovarian cancer in Taiwan: a case-control study in a low-incidence population. Gynecol Oncol. 2003;89(2):318-24.

59. Lauritzen C. On endocrine effects of oral contraceptives. Acta Endocrinol. Supl. 1968; Suppl 124:87-100.

60. Modan B, Hartge P, Hirsh-Yechezkel G, Chetrit A, Lubin F, Beller U, et al. Parity, oral contraceptives, and the risk of ovarian cancer among carriers and noncarriers of a BRCA1 or BRCA2 mutation. N Engl J Med. 2001;345(4):235-40.

61. Narod SA, Risch H, Moslehi R, Dorum A, Neuhausen S, Olsson $\mathrm{H}$, et al. Oral contraceptives and the risk of hereditary ovarian cancer. N Engl J Med. 1998; 339: 424-8.

62. Narod SA, Sun P, Ghadirian P, Lynch H, Isaacs C, Garber $\mathrm{J}$, et al. Tubal ligation and risk of ovarian cancer in carriers of BRCA1 or BRCA2 mutations: a case-control study. Lancet. 2001;357:1467-70.

63. Epithelial ovarian cancer and combined oral contraceptives. The WHO Collaborative Study of Neoplasia and Steroid Contraceptives. Int J Epidemiol. 1989;18(3):538-45.

64. Varma TR, Everad D, Hole D. Effect of natural estrogen on the serum level of follicle-stimulating hormone (FSH), estradiol and estrone in post menopausal women and its effect on endometrium. Acta Obstet Gynecol Scand. 1985;64:105-9.

65. Utian WH, Katz M, Davey DA, Carr PJ. Effect of premenopausal castration and incremental dosages of conjugated equine estrogens on plasma follicle-stimulating hormone, luteinizing hormone, and estradiol. Am J Obstet Gynecol. 1978;132:297-302.

66-Schiff I. The effects of conjugated estrogens on gonadotrofpins. Fertil Steril. 1980;33: 333-4.

67. Larsson-Cohn U, Johansson ED, Kagedal B, Wallentin L. Serum FSH, LH and estrone levels in postmenopausal 
patients on estrogen therapy. Br J Obstet Gynaecol. 1977;85: 367-72.

68. Bose CK. Does hormone replacement therapy prevent epithelial ovarian cancer? Reprod Biomed Online. 2005;11(1):86-92.

69. Mills PK, Riordan DG, Cress RD, Goldsmith DF. Hormone replacement therapy and invasive and borderline epithelial ovarian cancer risk. Cancer Detect Prev. 2005;29(2): 124-32.

70. Cooke ID, Anderton KJ, Lenton E. Hormone patterns at the climacteric. Postgr Med J. 1976; 52 Suppl 6: 12-6.

71. Irwin KL, Weiss NS, Lee NC, Peterson HB. Tubal sterilization, hysterectomy and subsequent occurrence of epithelial ovarian cancer. Am J Epidemiol. 1991;134:362-9.

72. Hankinson SE, Hunter DJ, Colditz GA, Willett WC, Stampfer MJ, Rosner B. Tubal ligation, hysterectomy, and risk of ovarian cancer. A prospective study. JAMA. 1993; 270:2813-8.

73. Slightler SE, Boike GM, Estape RE, Averette HE. Ovarian cancer in women with prior hysterectomy: a 14-year experience at the University of Miami. Obstet Gynecol. 1991; 78:681-4.

74. Parazinni F, Negri E, La Vecchia C, Restelli C, Franceschi S. Family history of reproductive cancers and ovarian cancer risk: an Italian case-control study. Am J Epidemiol. 1992;135(1):35-40.

75. Modugno F, Moslehi R, Ness RB, Nelson DB, Belle S, Kant JA, et al. Reproductive factors and ovarian cancer risk in Jewish BRCA1 and BRCA2 mutation carriers (United States). Cancer Causes Control. 2003;14(5):439-46.

76. Risch HA. Hormonal etiology of epithelial ovarian cancer, with a hypothesis concerning the role of androgens and progesterone. J Natl Cancer Inst. 1998;90:1774-86.

77. Helzlsouer KJ, Alberg AJ, Gordon GB, Longcope C, Bush TL, Hoffman SC. Serum gonadotrofins and steroid hormones and the development of ovarian cancer. JAMA. 1995;274:1926-30.

78. Motta ELA, Baracat EC, Haidar MA, Juliano I, Lima GR. Ovarian activity before and after gonadal suppression by GnRH-a in patients with polycystic ovary syndrome, hyperandrogenism, hyperinsulinism and acanthosis nigricans. Rev Assoc Med Bras. 1992;44(2):94-8

79. Paulson RJ. Fertility drugs and ovarian epithelial cancer: the endometriosis hypothesis. J Assist Reprod Genet. 1997;14:228-30.

80. Green A, Purdie D, Bain C, Siskind V, Russell P, Quinn M, et al. Tubal sterilization, hysterectomy and decreased risk of ovarian cancer. Survey of Womens's health Study Group. Int J Cancer. 1997;71:948-51.
81. Parazzini F, Negri E, La Vecchia C, Luchini L, Mezzopane R. Hysterectomy, oophorectomy, and subsequent ovarian cancer risk. Obstet Gynecol. 1993;81:363-6.

82. Modugno F, Ness RB, Allen GO, Schildkraut JM, Davis FG, Goodman MT. Oral contraceptive use, reproductive history, and risk of epithelial ovarian cancer in women with and without endometriosis. Am J Obstet Gynecol. 2004;191(3):733-40.

83. Blejer HP, Arlon R. Talc: a possible occupational and environmental carcinogen. J Occup Med. 1973;15:92-7.

84. Whittemore AS, Wu ML, Paffenbarger RS Jr, Sarles DL, Kampert JB, Grosser S, et al. Personal and environmental characteristics related to epithelial ovarin cancer. II. Exposures to talcum powder, tobacco, alcohol, and coffee. Am J Epidemiol. 1988;128(6):1228-40.

85. osenblatt KA, Szklo M, Rosenshein NB. Mineral fiber exposure and the development of ovarian cancer. Gynecol Oncol. 1992;45:20-5.

86. Cramer DW, Welch WR, Scully RE, Wojciechowki CA. Ovarian cancer and talc: a case-control study. Cancer. 1982;50:372-6.

87. Hartge P, Hoover R, Lesher LP, McGowan L. Talc and ovarian cancer. JAMA. 1983; 250:1844.

88. Harlow BL, Cramer DW, Bell DA, Welch WR. Perineal exposure to talc and ovarian cancer risk. Obstet Gynecol. 1992;80:19-26.

89. Qin LQ, Xu JY, Wang PY, Hashi A, Hoshi K, Sato A. Milk/ dairy products consumption, galactose metabolism and ovarian cancer: meta-analysis of epidemiological studies. Eur J Cancer Prev. 2005;14(1):13-9.

90. Larsson SC, Orsini N, Wolk A. Milk, milk products and lactose intake and ovarian cancer risk: a meta-analysis of epidemiological studies. Int J Cancer. 2006;118(2):431-41.

91. Akhmedkhanov A, Toniolo P, Jacquatte AS, Kato I, Koenig KL, Shore R. Aspirin and epithelial ovarian cancer. Prev Med. 2001;33(6):682-7.

92. Cramer DW, Harlow BL, Titus-ErnstoffL, Bohlke K, Welch WR, Greenberg ER. Over-the-counter analgesics and risk of ovarian cancer. Lancet. 1998;351(9096):104-7.

93. Fairfield KM, Hunter DJ, Fuchs CS, Colditz GA, Hankinson SE. Aspirin, other NSAIDs, and ovarian cancer risk (United States). Cancer Causes Control. 2002;13(6): 535-42.

94. Rodriguez-Burford C, Barnes MN, Oelschlager DK, Myers RB, Talley LI, Partridge EE, et al. Effects of nonsteroidal anti-inflammatory agents (NSAIDs) on ovarian carcinoma cell lines: preclinical evaluation of NSAIDs as chemopreventive agents. Clin Cancer Res. 2002;8(1):202-9. 